Liguo Yang*, Xin Wang, Dan Luo, Nana Liu and Dayong Tian

\title{
The crystal structure of
}

bis( $\mu_{2}-5$-chloro-2-oxido- $N$-(1-oxidoethylidene) benzohydrazonato- $\left.\mathrm{K}^{5} \mathrm{~N}, \mathrm{O}, \mathrm{O}^{\prime}: \mathrm{N}^{\prime}, \mathrm{O}^{\prime \prime}\right)$ hexkis(pyridine- $\kappa^{1} N$ )trinickel(II) - pyridine (1/1), $\mathrm{C}_{63} \mathrm{H}_{57} \mathrm{Cl}_{2} \mathrm{~N}_{13} \mathrm{Ni}_{3} \mathrm{O}_{6}$

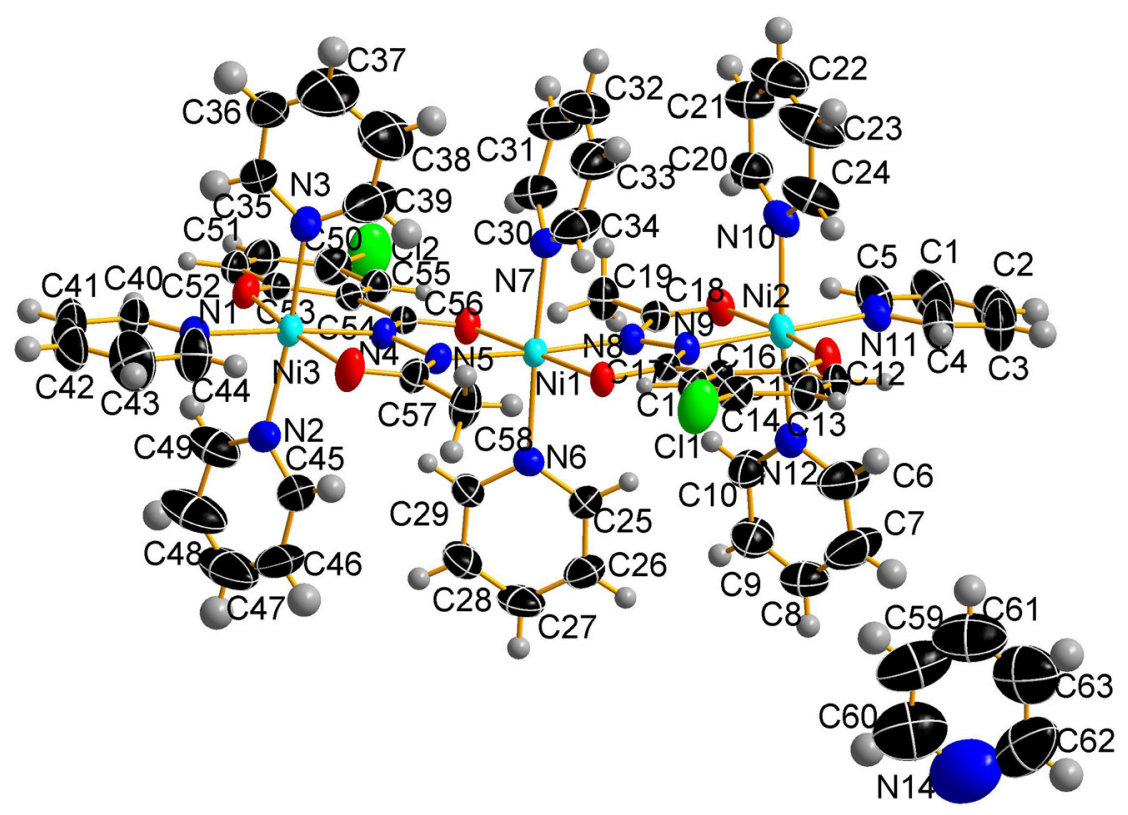

https://doi.org/10.1515/ncrs-2020-0600

Received November 6, 2020; accepted January 8, 2021; published online January 22, 2021

Table 1: Data collection and handling.

\begin{abstract}
$\mathrm{C}_{63} \mathrm{H}_{57} \mathrm{Cl}_{2} \mathrm{~N}_{13} \mathrm{Ni}_{3} \mathrm{O}_{6}$, monoclinic, $P 2_{1} / c, a=16.136(4) \AA$, $b=16.432(4) \AA, c=23.881(6) \AA, \beta=101.900(3)^{\circ}$, $V=6196(3) \AA^{3}, Z=4, R_{\mathrm{gt}}(F)=0.0461, w R_{\mathrm{ref}}\left(F^{2}\right)=0.1357$, $\mathrm{T}=298 \mathrm{~K}[1-3]$.
\end{abstract}

CCDC no.: 2054630

*Corresponding author: Yang Liguo, College of Chemistry and Environmental Engineering, Anyang Institute of Technology, Anyang, 455000, Henan, P. R. China, E-mail: Igyang@ayit.edu.cn. https:// orcid.org/0000-0003-4899-8298

Xin Wang, Dan Luo, Nana Liu and Dayong Tian, College of Chemistry and Environmental Engineering, Anyang Institute of Technology, Anyang, 455000, Henan, P. R. China

\begin{tabular}{ll}
\hline Crystal: & Red block \\
Size: & $0.06 \times 0.04 \times 0.02 \mathrm{~mm}$ \\
Wavelength: & Mo $K \alpha$ radiation $(0.71073 \AA)$ \\
$\mu:$ & $1.05 \mathrm{~mm}^{-1}$ \\
Diffractometer, scan mode: & Bruker APEX-II, $\varphi$ and $\omega$ \\
$\theta_{\text {max }}$, completeness: & $25.0^{\circ},>99 \%$ \\
$N(h k l)_{\text {measured }}, N\left(h k l l_{\text {unique }}, R_{\text {int }}:\right.$ & $72,238,10,897,0.053$ \\
Criterion for $I_{\text {obs }}, N(h k l)_{\text {gt }}:$ & $I_{\text {obs }}>2 \sigma\left(I_{\text {obs }}\right), 8298$ \\
$N(\text { param })_{\text {refined }}:$ & 786 \\
Programs: & Bruker $[1]$, SHELX $[2,3]$ \\
\hline
\end{tabular}

The molecular structure is shown in the figure. Table 1 contains crystallographic data and Table 2 contains the list of the atoms including atomic coordinates and displacement parameters. 
Table 2: Fractional atomic coordinates and isotropic or equivalent isotropic displacement parameters $\left(\AA^{2}\right)$.

\begin{tabular}{|c|c|c|c|}
\hline Atom & $x$ & $y$ & $z$ \\
\hline $\mathrm{Ni1}$ & $0.30718(2)$ & $0.39503(3)$ & $0.28225(2)$ \\
\hline $\mathrm{Ni} 2$ & 0.59164 (3) & $0.40468(3)$ & $0.39023(2)$ \\
\hline $\mathrm{Ni} 3$ & 0.02977 (3) & $0.33339(3)$ & $0.17469(2)$ \\
\hline 01 & $-0.03985(14)$ & $0.30837(16)$ & 0.23295 (11) \\
\hline 02 & $0.11658(16)$ & $0.36264(18)$ & $0.12628(10)$ \\
\hline 03 & $0.21115(14)$ & $0.37930(15)$ & $0.32435(10)$ \\
\hline 04 & $0.40322(13)$ & $0.41154(15)$ & $0.24046(9)$ \\
\hline 05 & 0.50154 (14) & 0.39447 (15) & $0.43821(10)$ \\
\hline 06 & 0.66494 (14) & $0.41634(17)$ & 0.33259 (11) \\
\hline N1 & $-0.07082(19)$ & $0.3139(2)$ & $0.10421(14)$ \\
\hline N2 & $-0.0134(2)$ & $0.4601(2)$ & $0.17227(16)$ \\
\hline N3 & $0.0633(2)$ & $0.2044(2)$ & $0.16906(15)$ \\
\hline N4 & $0.13045(16)$ & $0.35747(17)$ & $0.23514(12)$ \\
\hline N5 & $0.20385(16)$ & $0.37956(17)$ & $0.21484(12)$ \\
\hline N6 & $0.28788(18)$ & $0.52543(17)$ & $0.28177(12)$ \\
\hline N7 & 0.33262 (19) & $0.26500(18)$ & $0.28403(13)$ \\
\hline N8 & $0.41164(16)$ & $0.40238(17)$ & $0.35037(11)$ \\
\hline N9 & $0.48748(16)$ & 0.40517 (17) & $0.32985(12)$ \\
\hline N10 & $0.6033(2)$ & $0.2706(2)$ & 0.38994 (13) \\
\hline N11 & 0.69262 (19) & 0.40791 (19) & 0.46179 (14) \\
\hline N12 & 0.58868 (19) & $0.5387(2)$ & $0.39976(13)$ \\
\hline N14 & $0.7383(9)$ & $0.9771(6)$ & $0.5261(4)$ \\
\hline $\mathrm{Cl} 1$ & $0.57028(8)$ & $0.38651(9)$ & $0.08203(5)$ \\
\hline $\mathrm{Cl} 2$ & $0.04575(9)$ & $0.34987(11)$ & $0.48288(5)$ \\
\hline $\mathrm{C} 1$ & $0.7353(4)$ & $0.4230(4)$ & $0.5622(2)$ \\
\hline $\mathrm{H} 1$ & 0.7207 & 0.4226 & 0.5979 \\
\hline $\mathrm{C} 2$ & $0.8150(4)$ & $0.4379(4)$ & 0.5575 (3) \\
\hline $\mathrm{H} 2$ & 0.8563 & 0.4496 & 0.5899 \\
\hline $\mathrm{C} 3$ & $0.8353(3)$ & $0.4355(4)$ & $0.5046(3)$ \\
\hline H3 & 0.8907 & 0.4443 & 0.5004 \\
\hline $\mathrm{C} 4$ & $0.7715(3)$ & $0.4198(3)$ & $0.4575(2)$ \\
\hline $\mathrm{H} 4$ & 0.7850 & 0.4176 & 0.4215 \\
\hline C5 & $0.6755(3)$ & $0.4085(3)$ & $0.51376(18)$ \\
\hline H5 & 0.6201 & 0.3986 & 0.5174 \\
\hline $\mathrm{C} 6$ & $0.6535(4)$ & $0.5834(3)$ & $0.3923(3)$ \\
\hline $\mathrm{H} 6$ & 0.6998 & 0.5567 & 0.3832 \\
\hline $\mathrm{C} 7$ & $0.6572(4)$ & $0.6663(4)$ & $0.3971(4)$ \\
\hline $\mathrm{H} 7$ & 0.7054 & 0.6944 & 0.3923 \\
\hline $\mathrm{C} 8$ & $0.5890(4)$ & $0.7075(3)$ & $0.4090(2)$ \\
\hline $\mathrm{H} 8$ & 0.5878 & 0.7641 & 0.4103 \\
\hline $\mathrm{C} 9$ & $0.5239(3)$ & $0.6622(3)$ & $0.4186(2)$ \\
\hline $\mathrm{H} 9$ & 0.4771 & 0.6873 & 0.4282 \\
\hline $\mathrm{C} 10$ & $0.5265(3)$ & $0.5789(3)$ & $0.4141(2)$ \\
\hline $\mathrm{H} 10$ & 0.4809 & 0.5493 & 0.4217 \\
\hline $\mathrm{C} 11$ & $0.6387(2)$ & $0.4115(2)$ & $0.27738(15)$ \\
\hline $\mathrm{C} 12$ & $0.7005(2)$ & $0.4125(2)$ & $0.24263(17)$ \\
\hline $\mathrm{H} 12$ & 0.7571 & 0.4181 & 0.2605 \\
\hline $\mathrm{C} 13$ & $0.6807(2)$ & $0.4056(2)$ & 0.18399 (17) \\
\hline $\mathrm{H} 13$ & 0.7232 & 0.4068 & 0.1629 \\
\hline C14 & $0.5969(2)$ & $0.3968(2)$ & $0.15679(15)$ \\
\hline C15 & $0.5341(2)$ & $0.3977(2)$ & $0.18755(15)$ \\
\hline $\mathrm{H} 15$ & 0.4780 & 0.3929 & 0.1683 \\
\hline $\mathrm{C} 16$ & $0.5519(2)$ & $0.4055(2)$ & $0.24723(14)$ \\
\hline $\mathrm{C} 17$ & $0.4765(2)$ & 0.40798 (19) & $0.27399(14)$ \\
\hline C18 & $0.4272(2)$ & $0.3947(2)$ & 0.40645 (14) \\
\hline
\end{tabular}

Table 2: (continued)

\begin{tabular}{|c|c|c|c|c|}
\hline Atom & $x$ & $y$ & $z$ & $U_{\text {iso }} * / U_{\text {eq }}$ \\
\hline C19 & $0.3542(2)$ & $0.3838(3)$ & $0.43533(16)$ & $0.0482(10)$ \\
\hline $\mathrm{H} 19 \mathrm{~A}$ & 0.3551 & 0.3295 & 0.4504 & $0.072^{*}$ \\
\hline $\mathrm{H} 19 \mathrm{~B}$ & 0.3022 & 0.3924 & 0.4082 & $0.072^{*}$ \\
\hline $\mathrm{H} 19 \mathrm{C}$ & 0.3585 & 0.4223 & 0.4660 & $0.072^{*}$ \\
\hline $\mathrm{C} 20$ & $0.5614(3)$ & $0.2236(3)$ & $0.4204(2)$ & 0.0700 (13) \\
\hline $\mathrm{H} 20$ & 0.5267 & 0.2490 & 0.4417 & $0.084^{\star}$ \\
\hline $\mathrm{C} 21$ & $0.5665(4)$ & $0.1400(3)$ & $0.4222(3)$ & $0.0897(17)$ \\
\hline $\mathrm{H} 21$ & 0.5367 & 0.1100 & 0.4446 & $0.108^{*}$ \\
\hline $\mathrm{C} 22$ & $0.6163(5)$ & $0.1021(4)$ & $0.3905(3)$ & 0.099 (2) \\
\hline $\mathrm{H} 22$ & 0.6199 & 0.0456 & 0.3899 & $0.119 *$ \\
\hline $\mathrm{C} 23$ & $0.6608(5)$ & $0.1486(4)$ & $0.3595(2)$ & $0.102(2)$ \\
\hline $\mathrm{H} 23$ & 0.6962 & 0.1243 & 0.3382 & $0.122^{\star}$ \\
\hline C24 & $0.6525(4)$ & $0.2325(3)$ & $0.3603(2)$ & $0.0794(16)$ \\
\hline $\mathrm{H} 24$ & 0.6829 & 0.2636 & 0.3390 & $0.095^{*}$ \\
\hline $\mathrm{C} 25$ & $0.3531(3)$ & $0.5769(2)$ & $0.29261(18)$ & $0.0513(10)$ \\
\hline H25 & 0.4076 & 0.5555 & 0.3002 & $0.062^{*}$ \\
\hline C26 & $0.3437(3)$ & $0.6602(3)$ & $0.2931(2)$ & $0.0628(12)$ \\
\hline H26 & 0.3908 & 0.6940 & 0.3013 & $0.075^{*}$ \\
\hline $\mathrm{C} 27$ & $0.2636(3)$ & $0.6920(3)$ & $0.2812(2)$ & $0.0688(13)$ \\
\hline $\mathrm{H} 27$ & 0.2554 & 0.7480 & 0.2804 & $0.083^{*}$ \\
\hline $\mathrm{C} 28$ & $0.1960(3)$ & $0.6401(3)$ & $0.2704(2)$ & 0.0679 (13) \\
\hline $\mathrm{H} 28$ & 0.1410 & 0.6602 & 0.2629 & $0.081^{*}$ \\
\hline C29 & $0.2107(2)$ & $0.5574(2)$ & $0.27089(18)$ & $0.0508(10)$ \\
\hline H29 & 0.1644 & 0.5225 & 0.2632 & $0.061^{\star}$ \\
\hline C30 & $0.3130(3)$ & $0.2185(3)$ & $0.3245(2)$ & $0.0643(12)$ \\
\hline H30 & 0.2784 & 0.2397 & 0.3476 & $0.077^{\star}$ \\
\hline C31 & $0.3417(4)$ & $0.1395(3)$ & $0.3338(2)$ & $0.0885(18)$ \\
\hline H31 & 0.3275 & 0.1085 & 0.3630 & $0.106^{*}$ \\
\hline C32 & $0.3913(4)$ & $0.1077(3)$ & $0.2993(3)$ & $0.0870(17)$ \\
\hline H32 & 0.4099 & 0.0540 & 0.3038 & $0.104^{\star}$ \\
\hline C33 & $0.4126(4)$ & $0.1549(3)$ & $0.2590(3)$ & $0.096(2)$ \\
\hline H33 & 0.4480 & 0.1353 & 0.2359 & $0.115^{*}$ \\
\hline C34 & $0.3816(3)$ & $0.2328(3)$ & $0.2523(3)$ & 0.0768 (15) \\
\hline H34 & 0.3961 & 0.2647 & 0.2236 & $0.092^{*}$ \\
\hline C35 & $0.0100(4)$ & $0.1449(3)$ & $0.1628(4)$ & 0.128 (3) \\
\hline H35 & -0.0464 & 0.1573 & 0.1623 & $0.154^{\star}$ \\
\hline C36 & $0.0304(5)$ & $0.0634(4)$ & $0.1565(4)$ & $0.148(4)$ \\
\hline H36 & -0.0119 & 0.0240 & 0.1506 & $0.177^{\star}$ \\
\hline C37 & $0.1084(6)$ & $0.0431(4)$ & $0.1591(3)$ & $0.125(3)$ \\
\hline H37 & 0.1223 & -0.0089 & 0.1487 & $0.150^{*}$ \\
\hline C38 & $0.1715(5)$ & $0.1009(4)$ & $0.1779(4)$ & $0.117(2)$ \\
\hline H38 & 0.2284 & 0.0868 & 0.1890 & $0.140^{*}$ \\
\hline C39 & $0.1437(4)$ & $0.1809(4)$ & $0.1789(4)$ & $0.123(3)$ \\
\hline H39 & 0.1849 & 0.2213 & 0.1871 & $0.148^{*}$ \\
\hline $\mathrm{C} 40$ & $-0.1490(2)$ & $0.2981(3)$ & $0.1111(2)$ & 0.0599 (11) \\
\hline $\mathrm{H} 40$ & -0.1584 & 0.2933 & 0.1481 & $0.072^{*}$ \\
\hline C41 & $-0.2159(3)$ & $0.2888(3)$ & $0.0663(3)$ & $0.0801(16)$ \\
\hline H41 & -0.2697 & 0.2776 & 0.0728 & $0.096^{*}$ \\
\hline C42 & $-0.2033(4)$ & $0.2960(4)$ & $0.0122(3)$ & 0.0891 (19) \\
\hline H42 & -0.2481 & 0.2903 & -0.0190 & $0.107^{\star}$ \\
\hline C43 & $-0.1228(4)$ & $0.3118(4)$ & $0.0043(2)$ & 0.099 (2) \\
\hline $\mathrm{H} 43$ & -0.1120 & 0.3165 & -0.0323 & $0.118^{*}$ \\
\hline C44 & -0.0589 (3) & $0.3205(3)$ & $0.0515(2)$ & $0.0742(14)$ \\
\hline H44 & -0.0046 & 0.3315 & 0.0461 & 0.089 * \\
\hline C45 & $0.0266(4)$ & $0.5210(3)$ & $0.1542(3)$ & $0.108(2)$ \\
\hline H45 & 0.0755 & 0.5104 & 0.1406 & $0.129^{*}$ \\
\hline
\end{tabular}


Table 2: (continued)

\begin{tabular}{lrrrr}
\hline Atom & $\boldsymbol{x}$ & $\boldsymbol{y}$ & $\boldsymbol{z}$ & $\boldsymbol{U}_{\text {iso }}{ }^{*} \boldsymbol{U}_{\text {eq }}$ \\
\hline C46 & $-0.0022(6)$ & $0.6024(4)$ & $0.1548(4)$ & $0.148(4)$ \\
H46 & 0.0286 & 0.6445 & 0.1429 & $0.178^{*}$ \\
C47 & $-0.0733(7)$ & $0.6182(5)$ & $0.1723(4)$ & $0.151(5)$ \\
H47 & -0.0931 & 0.6713 & 0.1721 & $0.181^{*}$ \\
C48 & $-0.1167(6)$ & $0.5569(4)$ & $0.1905(3)$ & $0.117(3)$ \\
H48 & -0.1672 & 0.5665 & 0.2025 & $0.141^{*}$ \\
C49 & $-0.0836(4)$ & $0.4796(3)$ & $0.1906(2)$ & $0.0814(16)$ \\
H49 & -0.1124 & 0.4377 & 0.2046 & $0.098^{*}$ \\
C50 & $0.0205(3)$ & $0.3395(3)$ & $0.40822(17)$ & $0.0511(10)$ \\
C51 & $-0.0608(3)$ & $0.3196(2)$ & $0.38023(19)$ & $0.0528(10)$ \\
H51 & -0.1033 & 0.3119 & 0.4008 & $0.063^{*}$ \\
C52 & $-0.0779(2)$ & $0.3113(2)$ & $0.32200(18)$ & $0.0460(9)$ \\
H52 & -0.1331 & 0.2985 & 0.3038 & $0.055^{*}$ \\
C53 & $-0.0167(2)$ & $0.3211(2)$ & $0.28761(16)$ & $0.0375(8)$ \\
C54 & $0.0666(2)$ & $0.3437(2)$ & $0.31787(15)$ & $0.0347(8)$ \\
C55 & $0.0827(2)$ & $0.3518(2)$ & $0.37748(16)$ & $0.0419(9)$ \\
H55 & 0.1369 & 0.3658 & 0.3968 & $0.050^{*}$ \\
C56 & $0.1409(2)$ & $0.3606(2)$ & $0.29093(15)$ & $0.0324(7)$ \\
C57 & $0.1883(2)$ & $0.3806(2)$ & $0.15869(15)$ & $0.0406(8)$ \\
C58 & $0.2588(2)$ & $0.4047(3)$ & $0.13024(17)$ & $0.0580(12)$ \\
H58A & 0.2693 & 0.3617 & 0.1055 & $0.087^{*}$ \\
H58B & 0.3090 & 0.4148 & 0.1588 & $0.087^{*}$ \\
H58C & 0.2432 & 0.4532 & 0.1082 & $0.087^{*}$ \\
C59 & $0.6863(8)$ & $0.8488(6)$ & $0.5177(4)$ & $0.142(4)$ \\
H59 & 0.6393 & 0.8148 & 0.5138 & $0.171^{*}$ \\
C60 & $0.6740(8)$ & $0.9273(7)$ & $0.5182(4)$ & $0.151(4)$ \\
H60 & 0.6192 & 0.9478 & 0.5129 & $0.182^{*}$ \\
C61 & $0.7589(9)$ & $0.8161(6)$ & $0.5223(4)$ & $0.136(3)$ \\
H61 & 0.7640 & 0.7597 & 0.5220 & $0.163^{*}$ \\
C62 & $0.8171(9)$ & $0.9487(8)$ & $0.5301(4)$ & $0.151(4)$ \\
H62 & 0.8636 & 0.9832 & 0.5343 & $0.181^{*}$ \\
C63 & $0.8258(8)$ & $0.8616(7)$ & $0.5275(3)$ & $0.148(4)$ \\
H63 & 0.8788 & 0.8382 & 0.5295 & $0.178^{*}$ \\
\hline & & & &
\end{tabular}

\section{Source of material}

The educt $N^{\prime}$-acetyl-5-chloro-2-hydroxybenzohydrazide $\left(\mathrm{LH}_{3}\right)$, intended to be the bridging ligand in its deprotonated form was synthesized according to reference [4]. A mixture of $\mathrm{LH}_{3}(0.196 \mathrm{~g}, 1 \mathrm{mmol})$ and nickel acetate $(0.162 \mathrm{~g}, 1 \mathrm{mmol})$ and a few drops of a $\mathrm{NaOH}$ solution in $10 \mathrm{ml}$ methanol was stirred at room temperature for $1 \mathrm{~h}$. The mixture was filtered to remove impurties, and then left at room temperature. After a few days, red blocks of the ligand were formed. Yield $(0.23 \mathrm{~g}, 60 \%)$.

\section{Experimental details}

$\mathrm{H}$ atoms were geometrically placed $(\mathrm{C}-\mathrm{H}=0.95-0.98 \AA)$ and refined as riding with $U_{\text {iso }}(\mathrm{H})=1.2-1.5 U_{\text {eq }}(\mathrm{C})$ [3].

\section{Comment}

Pentadentate ligands have been synthesized in recent years [5]. Pentadentate ligands can react with the transition metals and rare earth metals [6, 7]. A lot of the ring structure containing complexes and metallacrown complexes have been synthesized. In recent years, our group synthesized several pentadentate ligands, the ligand $\mathrm{L} / \mathrm{LH}_{3}$ is one of them. The new ligand reacts with the transition metals [8].

As shown in the Figure, the title compound crystallizes in the monoclinic space group $P 2_{1} / c$ with four formula units in the unit cell. The bond lengths of $\mathrm{Ni}-\mathrm{O}$ are 2.030, 2.000, 2.035, 2.003 and 2.049 $\AA$, the bond lengths of $\mathrm{Ni}-\mathrm{N}$ are 2.081, $2.091,2.165,2.174,1.976,2.105,2.211,2.215,1.978,2.108,2.193$ and $2.198 \AA$, respectively, the bond lengths of $\mathrm{Ni}-\mathrm{N}$ from the pyridine molecule is longer than the bond lengths of $\mathrm{Ni}-\mathrm{N}$ from the anionic ligands, which are similar with the reference [8]. The trinuclear complex is almost centrosymmetric but this symmetry is not supported by the crystallographic findings. The Ni complex is formed by two chelated ligands three nickel ions and eight pyridine molecules. We can see that each nickel ion resides in a slightly distorted octahedral coordination environment, consisting of three pyridine $\mathrm{N}$, a phenolate $\mathrm{O}$, a carbonyl oxido $\mathrm{O}$ and an imine-type nitrogen of $\mathrm{L}$, which construct the five-membered and six-membered rings with each nickel atom.

Author contributions: All the authors have accepted responsibility for the entire content of this submitted manuscript and approved submission.

Research funding: Natural Science Foundation of Henan Province of China (Nos. 202300410010), Research Fund of Anyang Institute of Technology (Nos. YPY2019011, YPY2020025), Key discipline of environmental engineer of Anyang Institute of Technology.

Conflict of interest statement: The authors declare no conflicts of interest regarding this article.

\section{References}

1. Bruker. SMART and SAINT for Windows NT Software Reference Manuals, (version 5.0); Bruker Analytical X-Ray Systems: Madison, WI, 1997.

2. Sheldrick G. M. SADABS, A Software for Empirical Absorption Correction; University of Göttingen: Göttingen, Germany, 1997.

3. SHELXL Reference Manual, (version 5.1). Bruker Analytical X-Ray Systems: Madison, WI, 1997.

4. Yang M. X., Lin S., Chen L. J., Liu S. X. Synthesis and crystal structure of the trinuclear nickel(II) complex with Schiff base ligand $\mathrm{N}$ butylsalicylhydrazide. Chin. J. Inorg. Chem. 2003, 19, 434-436. 
5. Lin S., Liu S. X., Lin B. Z. Syntheses, crystal structures and magnetic property of the two $\mathrm{Mn}$ (II) 18-aza metallacrown-6 complexes. Chin. J. Inorg. Chem. 2002, 18, 1206-1210.

6. Lin S., Liu S. X., Lin B. Z. Synthesis, crystal structure and magnetic properties of a novel iron(III) 18-azametallacrown-6 compound. Inorg. Chim. Acta 2002, 328, 69-73.
7. Lin S., Liu S. X., Chen Z., Lin B. Z., Gao S. Synthesis, structure, and magnetism of a ferric 24-azametallacrown-8 complex. Inorg. Chem. 2004, 43, 2222-2224.

8. Chen X. H., Liu S. X. Syntheses and crystal structures of two nickel complexes with $\mathrm{N}$-substituted-salicyl ligand. Chin. J. Inorg. Chem. 2005, 21, 15-20. 